\title{
GGM Classifier with Multi-scale Line Detectors for Retinal Vessel Segmentation
}

\author{
Mohammad A. U. Khan · Tariq M. Khan · Syed S. Naqvi • M. Aurangzeb Khan
}

Received: date / Accepted: date

\begin{abstract}
Persistent changes in the diameter of retinal blood vessels may indicate some chronic eye diseases. Computerassisted change observation attempts may become challenging due to the emergence of interfering pathologies around blood vessels in retinal fundus images. The end result is lower sensitivity to thin vessels for certain computerized detection methods. Quite recently, multi-scale line detection method proved to be worthy for improved sensitivity towards lower-caliber vessels detection. This happens largely due to its adaptive property that responds more to the longevity patterns than width of a given vessel. However, the method suffers from the lack of a better aggregation process for individual line detectors. This paper investigates a scenario that introduces a supervised Generalized Gaussian mixture (GGM) classifier as a robust solution for the aggregate process. The classifier is built with class-conditional probability density functions as a logistic function of linear mixtures. To boost the classifier's performance, the weighted scale images are modeled as Gaussian mixtures. The classifier is trained with weighted images modeled on a Gaussian Mixture. The net effect is increased sensitivity for small vessels. The classifier's performance has been tested with three commonly available datasets: DRIVE, SATRE, and CHASE _DB1. The results of the proposed method (with an accuracy of 96\%,96.1\% and 95\% on DRIVE, STARE, and CHASE_DB1, respectively)
\end{abstract}

Mohammad A. U. Khan

Biometric Lab, Effat University Research and Consultancy Institute, Jeddah, Saudi Arabia

Tariq M. Khan, Saud S. Naqvi

Department of Electrical and Computer Engineering, COMSATS University Islamabad, Islamabad Campus, Pakistan

School of Computing and Communication, Infolab21, Lancaster University, Lancaster, LA1 4WA, United Kingdom demonstrate its competitiveness against the state-of-the-art methods and its reliability for vessel segmentation.

\section{Introduction}

The contraction and expansion of blood vessels, particularly in small arteries, enable organs to adjust their own blood flow to meet the metabolic requirements of the tissue. Though variation in blood vessel diameter is part of the natural control of blood flow in healthy subjects [1], the sustained changes may indicate the presence of pathologies. The pathologies like micro-aneurysm, exudate, make it difficult to analyze blood vessels around the foveal avascular zone (FAZ) region. Tracking Vessel diameter changes for a longer period of time is therefore of interest to better understand the regulation of blood flow [2,3] and are helpful in prediction, diagnosis or progression of certain diseases like Diabetic Retinopathy, Glaucoma and leakages [4-7]. There are a number of different modalities to observe blood vessels such as Computer Tomography (CT) scans, Magnetic Resonance Imaging (MRI), X-rays, and Fundus Photography (used here) [8,9].

In the clinical setting, fundus retinal images are acquired as color images composed of red, green and blue channels. The inverted green channel is then generally selected for further processing as its gray-scale representation as it provides the highest background vessel contrast among other channels. Vessels appear with higher intensity levels (white) and the background region with low intensity (black).

Retinal image analysis plays an important role in diagnosing eye-related diseases, the largest of which is Diabetic Retinopathy. Diabetic patients are more suspectable to develop eye diseases such as cataracts and glaucoma in the longer run, however, the impact on the retina is the most threatening that can ultimately lead to vision loss. Diabetic 
Retinopathy (DR) damages retinal vessels.

The emphasis of a computerized retinal analysis system is on the automatic extraction of the vasculature. In literature, there are two types of automated segmentation techniques: supervised and unsupervised techniques. Supervised techniques are the ones where segmentation algorithm learns from labeled data; once the classifier has learned from the data, the process of segmentation can be applied to the test data. On the contrary, unsupervised techniques operate without any learning schemes. Instead, they learn to segment based on certain rules developed due to domain knowledge. In general, the supervised methods always provide better performance over those of unsupervised ones. However, supervised methods are in need of training data which is often not available in case of screening programs and large population-based studies. Furthermore, the supervised method lacks the necessary generalization to reduce the mismatch in performance when dealing with unknown test data.

Though monumental work has been reported for supervised and unsupervised retinal vessel extraction, the lowcontrast vessels are still being elusive. The focus of the research presented in this paper is to capture low-contrast narrow vessels with a supervised method that can work with a limited labeled dataset, leading to an improved accuracy of the vascular segmentation process. To achieve this, a Generalized Gaussian mixture (GGM) classifier is proposed as an appropriate solution to the aggregation problem encountered in multi-scale line detection methodology. The application of this supervised detector is not limited to vessels detection only, it can be used in other applications such as line detection in visual tracking [10-15] as well.

This paper is organized as follows. In Section 2, related work is presented. In Section 3 proposed method is described. Section 4 discusses the experimental results followed by the last section of the conclusion.

\section{Related Work}

A number of successful computer-assisted analysis reported in the literature with the goal of separating vessel from non-vessel regions. However, both the visual inspection and objective assessment of the classification appears to have some performance gaps. The largest hindrance is the inability of the automatic method to capture some of the thinnest vessels that are barely perceivable even by the keen human observers. A recently introduced line detector provides an improved sensitivity towards thin vessels [16] due to its peculiar one-pixel-wide line shape. The action of line detectors follows a difference equation of the form: $R(x, y ; W)=\max _{0 \leq \theta \pi} I_{a}(x, y ; W, \theta)-I_{i}(x, y ; W)$, where $I_{i}(x, y ; W)$ is the average value calculated with an isotropic $W \times W$ local window surrounding the pixel, and $I_{a}(x, y ; W, \theta)$ is anisotropic average computed with pixels directly under the line of length $W$, centered at pixel in a specified direction $\theta$. In their basic format, the length is kept same as that of window size $W$. The line detectors were later generalized in [17], by allowing the flexibility of changing line length within a large window size, and thus result in, what they claimed as a multi-scale line detector. A popular supervised method is a morphology-based detector that is used with a Gaussian mixture model to classify some hard-to-detect pixels in [18]. Khan et. al. [9] applied scalenormalized detector for vessels. This method can detect vessels regardless of their sizes. Soomro et. al. [6] proposed an ICA-based enhancement technique for retinal vessels segmentation. The low and varying contrast problem has been tackled using Independent Component Analysis (ICA).

In addition to the Gaussian Mixture model approach, some other popular supervised methods including Neural networks (NN) have been rigorously applied in the recent past to the retinal vessel segmentation problem [19]. Pertinent to mention is the NN method presented in [20], where classifier trained on one dataset, later tested on unseen images of another dataset provided fairly accurate results in terms of area under the receiver operating curves (AUC). Recent studies have investigated deep neural networks (DNN) for retinal vessel segmentation [21-23]. Fu et. al. [24] utilize the fully convolutional neural network (CNN) to generate a vessel probability map. For providing the long-range interactions between pixels and discriminative vessel probability map Conditional Random Fields (CRFs) are used. These neural network methods though provided much-needed accuracy, their generalized abilities are critically limited when used with small sized data. Therefore, we opted to use the generalized Gaussian model (GGM), for its better generalization property for small data sets, in this work.

\section{Proposed Method}

A multi-scale line detector for a given image operates by creating a series of scaled images as $S_{L}(x, y ; L)=$ $\max 0 \leq \theta \leq \pi S_{L}(x, y ; L, \theta)$, where $L=L_{i}, \ldots, L_{f}$. The need for adopting the multi-scale methodology arises due to the observation that basic line detectors produce unwanted shadows in proximity to strong vessels. However, with a multi-scale framework the new issue of aggregation appears. One straightforward way is to arithmetically average images obtained at various scale $S(x, y ; L)$ to get the final output, $G(x, y)=\frac{1}{n} \sum_{L=L_{i}}^{L_{f}} S_{L}(x, y ; L)$, where $n$ represents the count of scaled responses, an idea suggested in [17]. Aggregate response computed in this way deals fairly well with two prominent problems associated with the response of line detectors. First is the dominance of background noise due to small-scale line detectors, and the second is the presence of shadows in proximity to strong vessels in large-scale responses. However, scaled images are computed incurring different precision errors, and thus should be weighted differently based on their noise profile. Later on, in a subse- 
quent research, the authors [25] floated an idea of weighted linear combination instead of a simple addition to mitigate unequal contribution issues with individual line detectors, that is, $f(x, y)=\frac{1}{n} \sum_{L=L_{i}}^{L_{f}} w_{L} S(x, y ; L)$, where $w_{L}$ is the weight assigned to a scaled response due to line detector of length $L$. However, the author fell short of specifying a systematic method to compute these weights, ad-hoc weights in the form of increasing index were used in their work. We proposed the use of weights that are inversely related to noise variance of a given scaled image in this work.

This paper presents a semi-supervised weighted multiscale line detector, where supervision is performed for the aggregation of multi-scale line detector outputs for weight learning. A small amount of labeled data is found adequate to train the model to capture the distribution of the data and later the model can be easily generalized by the inclusion of new test images. The lower amount of supervision, required only at the multi-scale aggregation stage, makes the proposed method much more attractive for screening programs used in large population studies, where there is a scarcity of labeled data. Fig. 1 shows the block diagram of the proposed method.

\subsection{Supervised weighted multi-scale line detector}

Fundus camera provides color retinal images, in the form of three basic channels, red, green, and blue. We specifically worked with green channel only, whose inverted image is used as a gray-scale representation, where vessels have the white intensity and the non-vessel region is predominate of black color. The reason for choosing the green channel is the factor that it provides the highest contrast for the blood vessels among other channels [26]. By varying line lengths $L$ within a large window size $W$, a series of line detectors masks, from small to large, are generated. The line masks are then convolved with the input to produce a series of scaled images $S(x, y ; L)$. Now since $n$ images of the same input are available, then smoothing can be accomplished without blurring excessively by arithmetic averaging as. $G(x, y)=\frac{1}{n} \sum_{k=1}^{n} S(x, y: k)$. However, due to the difference in intensity dynamic range associated with each of the scale images, they have to be first standardized to have zero mean and unit variance and then added pixel-wise. This addition results in lesser noise and clearer vessel tree structure, that can later be converted to binary output with an appropriate threshold to results in the white foreground for vessels and black background for rest of the image. The multi-scale line detection framework was found to provide an improved classification as compared other detectors [17]. The Fig. 2 shows the scale- 3 and scale-13 images for a test image, related to a publicly available DRIVE database. The histograms of the vessel and the non-vessel region are depicted due to the availability of ground truth for the test im- ages. We clearly see a large overlap in their histogram, making it difficult to find a threshold. However, the encouraging sign is that both classes depict normal distribution behavior, that can later be used to the advantage of adopting a stable Generalized Gaussian modeling (GGM) approach for both classes.

The performance of multi-scale line detection for retinal images can be improved by making use of labeled training set, resulting in supervised classification. Towards that end, a retinal image is thought of made with two classes, the vessel class (foreground) and the non-vessel class (background). Each class $C_{1}$ (vessels) and $C_{2}$ (nonvessels), is in turn represented as logistic function of linear combination of scale images $S_{L}(x, y)$, known as predictors. Specifically, for the case of vessel class, it is described as $C_{1}=\frac{1}{1+\exp ^{-\left(\beta_{0}+\beta_{1} S_{1}+\beta_{2} S_{2}+\cdots+\beta_{n} S_{n}\right)}}$ The parameter set $\beta_{0}, \beta_{1}, \beta_{2}, \cdots, \beta_{n}$ is computed using Expected Minimization with maximum likelihood function for the labeled training set belonging only to the vessel class. In case of linear regression, the closed-form solution for the optimal parameters can be found. However, this is not the case for logistic regression, where some iterative fitting procedure is incorporated that needs good convergence properties. As our scaled images show normal distribution for both classes, the convergence of training method is achieved with fewer iteration steps. For assessing a test image pixel to be one of the two opposite classes, the vessels or non-vessels, Bayes rule is applied, which is $P\left(C_{i} \mid v\right)=\frac{p\left(v / C_{i}\right) P\left(C_{i}\right)}{p(v)}$, where $p\left(v / C_{i}\right)$ is the class-conditional probability density function, $P\left(C_{i}\right)$ is the prior probability of class $C_{i}$, and $p(v)$ is the probability density function of $\mathrm{v}$ (sometimes called evidence). The class-conditional probabilities, also referred to as likelihood, are represented as linear combination of Gaussian functions:

$p\left(v \mid C_{i}\right)=\frac{1}{1+\exp ^{-\left(\beta_{0}+\beta_{1} p\left(v \mid S_{1}, C_{i}\right)+\beta_{2} p\left(v \mid S_{2}, C_{i}\right)+\cdots+\beta_{n} p\left(v \mid S_{n}, C_{i}\right)\right)}}$

, where $p\left(v \mid S_{j}, C_{i}\right)$ is the Gaussian distribution and $\beta_{j}$ are the parameters to be estimated from labeled training set. Once trained, the class-conditional probabilities along with priors are used to find the posterior probability for a given test pixel to be in a vessel class.

Due to large number of pixels, in our all experiments, two million pixel within FOV are randomly chosen to train the classifiers. For the GGM classifier, the number of predictors is same as that of scaled image count for modeling both vessel and non-vessel classes. The convergence while choosing parameters was fast, within a couple of iterations. GGM supervised classification is also tested with weighted scale images. Admitting the fact that scale images contribute less noise as we move towards larger scales, as shown in Fig.2(b), scale images are first weighted with the inverse of their noise variances and then fed for logistic regression modeling, resulting in a different set of parameters 


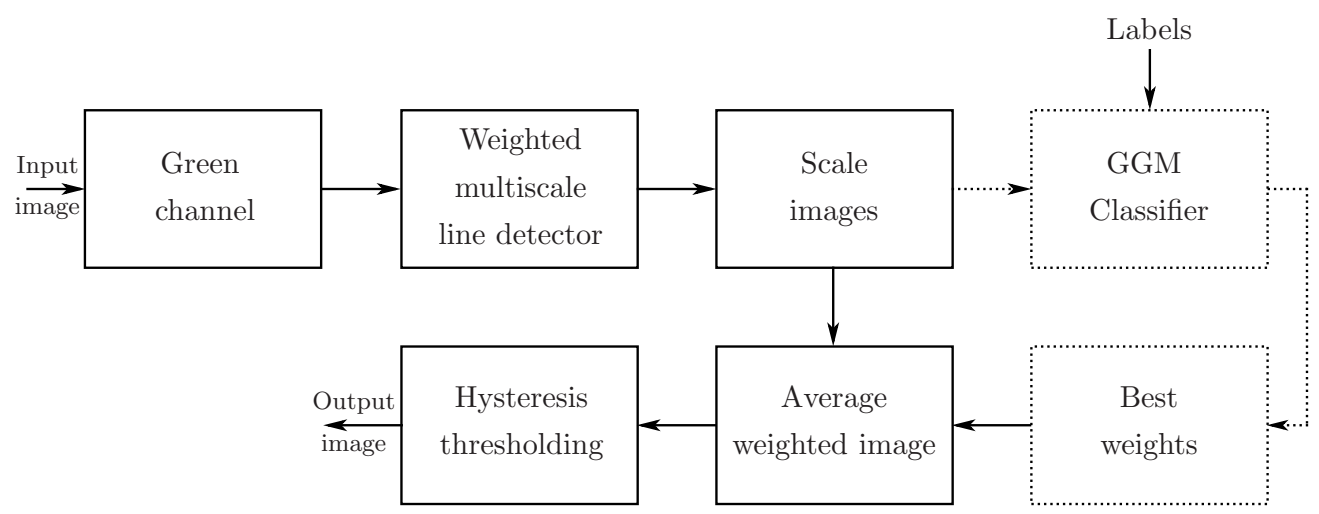

Fig. 1 Block diagram of the proposed method. Dotted blocks/lines are for training purposes, and blocks/lines are for testing purposes.

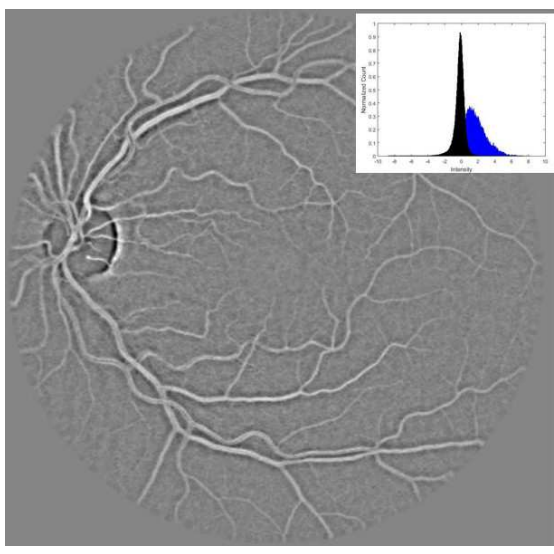

(a)

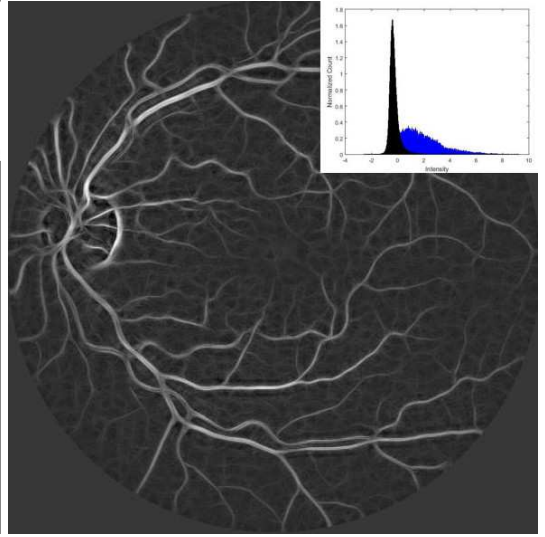

(b)

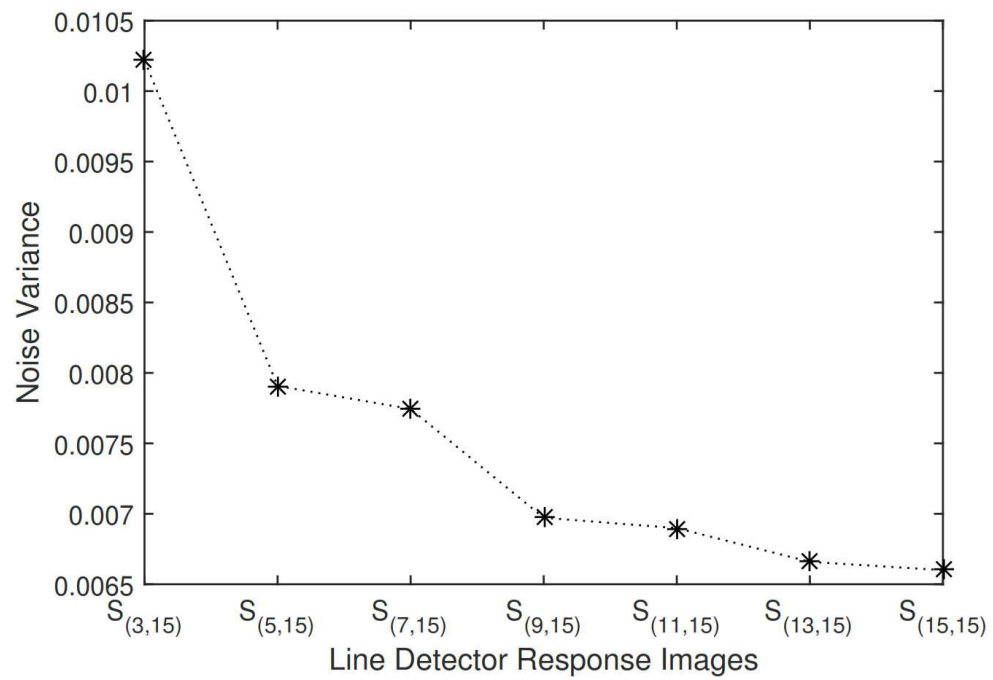

(c)

Fig. 2 The Fig shows on the left two scale images, one due to line detector of scale 3, and other for scale-13. Also, depicted the vessel and non-vessel histogram, showing large overlap for both images. The graph on right is the depiction of fact that scale images have noise variance that decreases with scale Graph.

for GGM classifier. The testing procedure is also used with the weighted scale images of the test image. We refer to this scheme as supervised classification with weights.

The output of the classifier is a posterior probability image for the given test input image. The next task is to find a threshold to convert it into a binary image. Though we see a clear improvement in the sense that the overlap area between two classes is reduces as a result of supervised classification, the minimum overlapping is still there. To clear this minimum overlap, a threshold strategy has to be adopted 
with some heuristics. To find an appropriate threshold to pick thin vessels, one way is to adopt a Shannon-entropy based threshold scheme. The first threshold $T_{1}$ is calculated by finding the maximum of Shannon entropy curve, and all the pixels of the image above that threshold are clipped to that threshold value. Now another Shannon entropy curve is found for this clipped image and we compute the second threshold $T_{2}$. All the pixels below that threshold are clipped to that value. The doubled-clipped image is now presented for the third time to Shannon entropy curve, and third threshold $T_{3}$ is obtained, which is our final threshold that can be used for producing all binary results. It was found in our test results, that the entropy-scheme is severally effected with non-uniform illumination present in an image. Therefore, we inclined to adopt a morphological technique with hysteresis, as described in next section.

\section{Thresholding using Hysteresis}

Thresholding techniques are based on image characteristics i.e. image space region. The multilevel image is converted into a binary image by thresholding. Thresholding is usually categorized into two types: local thresholding and global thresholding. Fixed threshold (T) approach refers to global thresholding and variable threshold approach is called local thresholding. The global thresholding is likely to flop in case of uneven illumination of background.

There are a couple of issues with a single threshold. First, single threshold takes into account intensity with no regard to connectivity among pixels. Therefore, there is no guarantee that the process will provide contiguous areas. Second, changes in illumination across the scene may cause the single threshold to miss some parts of the vessels being darker. Third, a single threshold is quite sensitive; changing threshold value by even a small amount can have a large impact on the binary output. Therefore, a need arises to add flexibility to a single threshold.

In this paper, a band of thresholds, as implied by hysteresis threshold process, are used. Hysteresis threshold consistently outperforms single threshold techniques [27] and is now a de facto standard in Canny edge detection [28]. The process is used for linking strong points with weak points caring vessel path tracing. A popular way to implement hysteresis threshold is via morphological operation (openingby-reconstruction). It enables the objects (vessels) to be exactly restored to their original shape initiated with seed points. The method requires two images, known as a marker (A) and mask (M). A mask is a binary image that provides boundaries for the growing process to stop. It is obtained from the combined output by using threshold $T_{L}$. Whereas the marker image contains seed points and is found as a result of threshold $T_{H}$. By defining a $3 \times 3$ structuring element $B$ for connectivity, the growing procedure can be described by iteratively applying

$A_{n+1}=\left(A_{n} \oplus B\right) \cap M$ and ultimately stopping when $A_{n+1}=A_{n}$. Here $\oplus$ is the exclusive-OR operation and $\cap$ is an intersection operation.

The binary output images obtained through hysteresis threshold method are having tiny holes inside vessel tree structures with some breaks at the junction points. To fill the gaps, the morphological dilation process is adopted. Dilation is one of the two basic operators in the field of mathematical morphology. The basic impact of the operator on a binary image is to gradually enlarge the boundaries of regions of foreground pixels (i.e. white pixels, typically). Thus areas of foreground pixels grow in size while holes within those regions become smaller.The dilation operator takes two pieces of data as inputs. The first is the image which is to be dilated. The second is a set of coordinate points known as a Structuring Element. It is this structuring element that determines the precise effect of the dilation on the input image. However, not to disturb the tree structure, a small linear structuring element, on the tune of only 3 pixels long, is utilized. First, four $3 \times 3$ templates of structuring element are created to go along 0 degrees, 45 degrees, 90 degrees, and 135 degrees. Then, the binary image is dilated with all the four templates, to produce four dilated images corresponding to four unique directions. Here, we take the help of Kas and Witkin [29] method to generate a reliable directional field, which produces directional image $\theta(x, y)$ for the input binary image. The directional image values are quantized to only four directions to match with template directions. Next, based on directional image quantized values, we pick the appropriate dilated image values to form a new image, called final binary image, as shown in Fig. 3(c).

\section{Result and Discussion}

The proposed modeling is evaluated on three publicly available databases DRIVE, STARE and CHASE_DB1. The DRIVE database provides 40 retinal images with their ground truth data. The first twenty images are used as training set and the rest as testing set for producing the objective assessment. STARE data set contains 20 images and there is no separate training and test set available. In literature two techniques random sample and leave-one-out are available [30]. In this paper leave-one-out technique is used for training [31], in which each image is tested using a trained model on the other 19 images. The CHASE_DB1 data set contains 28 images. First 20 images are used for training and last 8 images are used for testing.

The performance is quantified by comparing a segmented output image with its corresponding golden-truth image (a manually segmented image) available at the database. Three parameters, sensitivity, specificity, and accuracy, are calculated for measuring the performance. Four measures are required for the calculation of these three parameters. These four measures are true positive (TP), true negative (TN), false positive (FP) and false negative (FN). The TP represents the number of vessels correctly identified 


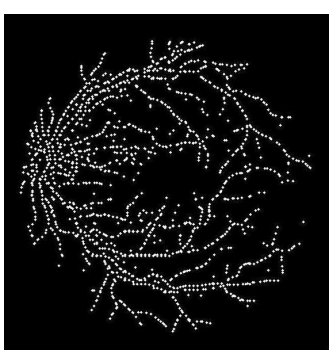

(a)

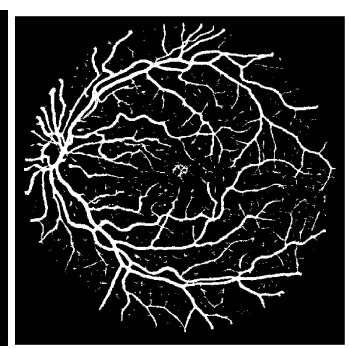

(b)

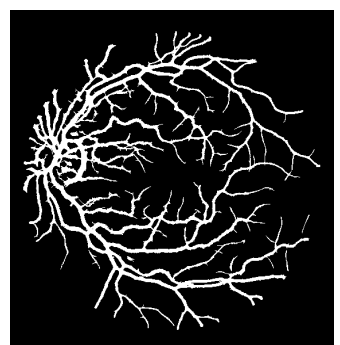

(c)

Fig. 3 a) Marker Image. b) Mask Image. c) Final binary result.

while TN represents the number of non-vessels correctly identified. The FP represents the number of non-vessels wrongly identified as vessels while FN represents the number of vessels wrongly identified as non-vessels.

The accuracy can be defined as sum of correctly identified vessels and non-vessels to the sum of total number of pixels,

Accuracy $=\frac{T P+T N}{T P+F P+T N+F N}$.

The ratio of correctly identified vessels and the total number of vessel is called sensitivity,

Sensitivity $=\frac{T P}{T P+F N}$.

The ratio of correctly detected vessels and total number of non-vessels is called specificity,

Specificity $=\frac{T N}{T N+F P}$.

For visual inspection, the Fig. 4 provides the images for multi-scale line detection, supervised multi-scale, and supervised multi-scale with weights. The supervised classification shows more sensitivity to thin vessels.

5.1 Comparison of performance measures with other algorithms

Several approaches are selected for comparison, both supervised and unsupervised. For performance analysis three publicly available databases DRIVE, STARE and CHASE_DB1 are used. The results on the two data sets are shown in Table 1 and the results of CHASE_DB1 are shown in Table 2. On DRIVE datebase, proposed method's sensitivity is 0.782 which is slightly less than Orlando et al. [33] sensitivity 0.753 . While on STARE its sensitivity is 0.786 which is slightly greater than Soomro et al. [32] sensitivity 0.785 . The sensitivity of the proposed method is higher than as compared to other methods and is presented in Table 1 for STARE data set.

Specificity (Sp) of the proposed method is comparable to the state-of-art methods on these two data sets. On DRIVE dataset, proposed method's specificity is higher than all existing methods while on SATRE database it is slightly higher than Soomro et al. [7] Accuracy (AC) is the mean measurement of closeness to the interpreted standards. The accuracy of the proposed method is also better than state-of-art methods presented in Table 1 on both data sets. On STARE database, the accuracy of the propose method is slightly higher than Soomro et al. [7]. On CHASE-DB1 database, the performance of proposed method is better than other two methods presented in Table 2. The sensitivity of the proposed method is 0.7626 , specificity is 0.9717 and accuracy is 0.9507 , respectively.

\section{Conclusion}

Several eye diseases can be diagnosed by observing variations in retinal blood vessel diameters. It is necessary to detect accurate retinal vessels. Accurate detection of vessels is a challenging task in the presence of noise and pathologies. In the literature, many approaches are proposed to extract retinal blood vessels. This paper provides empirical evidence of improved aggregate process linked with multi-scale line detectors using inverse-noise variances based predictors having generalized Gaussian mixture behavior. The lower amount of supervision required at the multi-scale aggregation makes the proposed method more suitable for screening programs and large population studies, where there is scarcity of labelled data. Another advantage of the proposed approach is its fast training time which is order of magnitude faster than the deep neural networks counterpart. The weight learning process becomes a simplified task with the use of GGM classifier. On the other hand, deep neural network classifier has shown the ability to provide comparable results to the GGM, but at a larger computational cost for its training phase. The visual comparison of the approaches provides evidence towards improved sensitivity for thin vessel detection.

\section{Acknowledgement}

The authors would like to thank Effat University in Jeddah, Saudi Arabia, for funding the research reported in this paper through the Research and Consultancy Institute. 


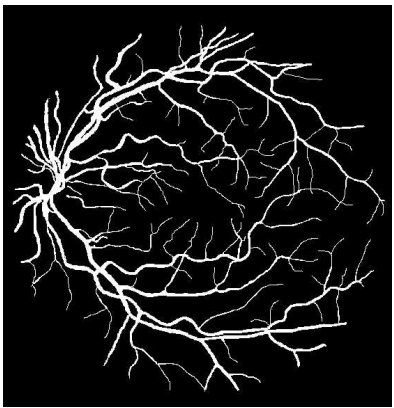

(a)

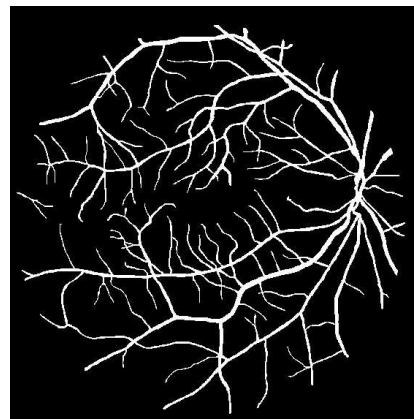

(e)

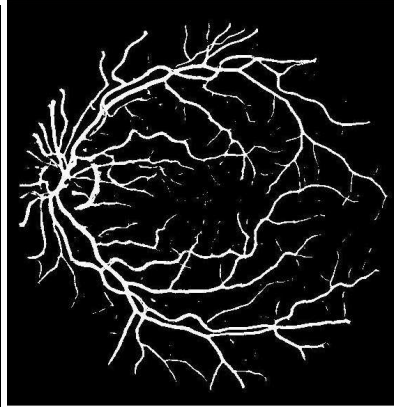

(b)

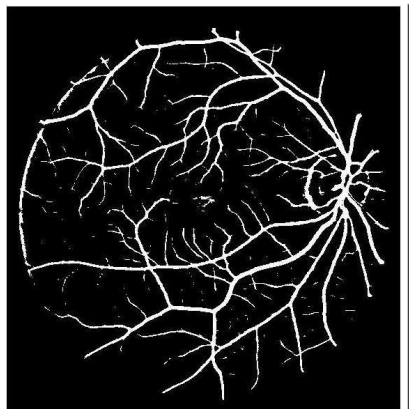

(f)

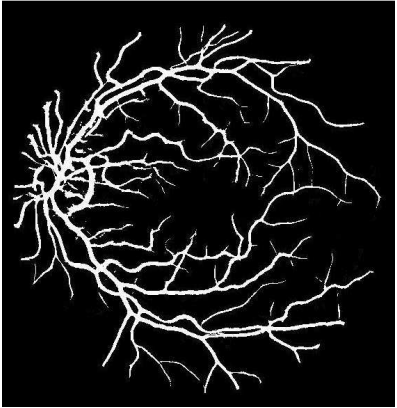

(c)

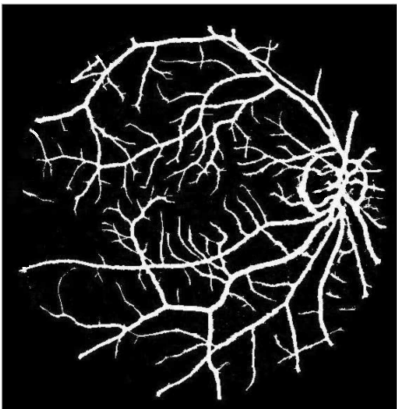

(g)

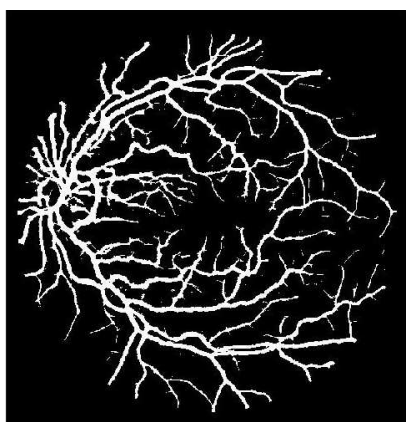

(d)

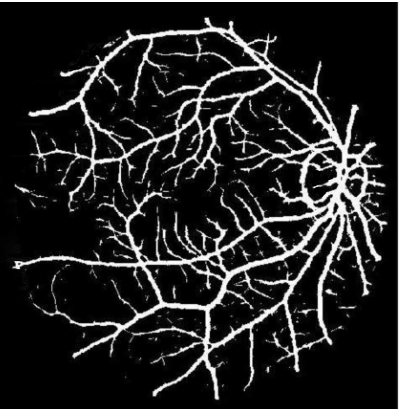

(h)

Fig. 4 Coulumn one show the ground truth images. Column two shows multiscale line detection method applied on the 1st and 16th image of DRIVE database. Column three shows proposed multiscale line detection without weights is applied on the 1st and 16th image of DRIVE database. Column four shows proposed multiscale line detection with weights is applied on the 1st and 16th image of DRIVE database.

Table 1 Performance Analysis of Segmentation Model

\begin{tabular}{|c|c|c|c|c|c|c|}
\hline \multirow[b]{2}{*}{ Methods } & \multicolumn{3}{|c|}{$D R I V E$} & \multicolumn{3}{|c|}{$S T A R E$} \\
\hline & Se & $S p$ & $A C$ & $\mathrm{Se}$ & $S p$ & $A C$ \\
\hline \multicolumn{7}{|l|}{ Unsupervised Methods } \\
\hline Nguyen et al. [17] & - & - & 0.940 & - & - & 0.932 \\
\hline Soomro et al. [34] & 0.713 & 0.968 & 0.941 & 0.711 & 0.965 & 0.942 \\
\hline Khan et al. [35] & 0.734 & 0.967 & 0.951 & 0.736 & 0.971 & 0.95 \\
\hline Soomro et al. [7] & 0.753 & 0.976 & 0.943 & 0.784 & 0.981 & 0.961 \\
\hline \multicolumn{7}{|l|}{ Supervised Methods } \\
\hline Steal et al. [31] & - & - & 0.946 & - & - & 0.951 \\
\hline Soares et al. [36] & - & - & 0.946 & - & - & 0.948 \\
\hline Lupascu et al. [37] & 0.720 & - & 0.959 & - & - & - \\
\hline Orlando et al. [33] & 0.785 & 0.967 & - & - & - & 0.951 \\
\hline Liskowski [22] & - & - & 0.949 & - & - & 0.949 \\
\hline Proposed Method & 0.782 & 0.986 & 0.960 & 0.796 & 0.983 & 0.961 \\
\hline
\end{tabular}

Table 2 Performance comparison on CHASE_DB1 database

\begin{tabular}{llll}
\hline & Se & Sp & AC \\
\hline $\begin{array}{c}\text { Unsupervised } \\
\text { Azzopardi [38] }\end{array}$ & 0.7585 & 0.9587 & 0.9387 \\
\hline Supervised & & & \\
Fraz [19] & 0.7224 & 0.9711 & 0.9469 \\
Proposed & $\mathbf{0 . 7 6 2 6}$ & $\mathbf{0 . 9 7 1 7}$ & $\mathbf{0 . 9 5 0 7}$
\end{tabular}

\section{References}

1. Knudtson MD, Klein BEK, Klein R, Wong TY, and Hubbard LD, "Variation associated with measurement of retinal vessel diameters at different points in the pulse cycle," J Ophthalmol, vol. 88, pp. 57-61, 2004.

2. Fischer JG, Mewes H, Hopp HH, and Schubert R, "Analysis of pressurized resistance vessel diam- eter changes with a low cost digital image processing device," Comput Meth Prog Bio, vol. 50, pp. 23-030, 1996.
3. Tyml K, Anderson D, Lidington D, and Ladak HM, "A new method for assessing arteriolar diameter and hemodynamic resistance using image analysis of vessel lumen," Am J Physiol Heart Circ Physiol, vol. 284, pp. H17218, 2003.

4. Patton N, Aslam T, Macgillivray T, Pattie A, and Deary IJ, "Retinal vascular image analysis as a potential screening tool for cerebrovascular disease: a rationale based on homology between cerebral and retinal microvasculatures.," J Anat, vol. 206, pp. 319-348, 2005 .

5. Wang JJ, Liew G, Klein R, Rochtchina E, and Knudtson MD, "Retinal vessel diameter and cardiovascular mortality: pooled data analysis from two older populations.," European Heart Journal, vol. 28, pp. 19841992, 2007.

6. Toufique Ahmed Soomro, Tariq Mahmood Khan, Mohammad AU Khan, Junbin Gao, Manoranjan Paul, and Lihong Zheng, "Impact of ica-based image enhancement technique on retinal blood vessels segmentation," IEEE Access, vol. 6, pp. 3524-3538, 2018. 
7. Toufique Ahmed Soomro, Junbin Gao, Tariq Khan, Ahmad Fadzil M Hani, Mohammad AU Khan, and Manoranjan Paul, "Computerised approaches for the detection of diabetic retinopathy using retinal fundus images: a survey," Pattern Analysis and Applications, vol. 20, no. 4, pp. 927-961, 2017.

8. Mohammad AU Khan, Tariq M Khan, DG Bailey, and Toufique A Soomro, "A generalized multi-scale line-detection method to boost retinal vessel segmentation sensitivity," Pattern Analysis and Applications, pp. 1-20, 2018.

9. Mohammad AU Khan, Tariq M Khan, Toufique Ahmed Soomro, Nighat Mir, and Junbin Gao, "Boosting sensitivity of a retinal vessel segmentation algorithm," Pattern Analysis and Applications, pp. 1-17, 2017.

10. X. Lan, S. Zhang, P. C. Yuen, and R. Chellappa, "Learning common and feature-specific patterns: A novel multiple-sparserepresentation-based tracker," IEEE Transactions on Image Processing, vol. 27, no. 4, pp. 2022-2037, 2018.

11. X. Lan, A. J. Ma, and P. C. Yuen, "Multi-cue visual tracking using robust feature-level fusion based on joint sparse representation," in 2014 IEEE Conference on Computer Vision and Pattern Recognition, 2014, pp. 1194-1201.

12. X. Lan, A. J. Ma, P. C. Yuen, and R. Chellappa, "Joint sparse representation and robust feature-level fusion for multi-cue visual tracking," IEEE Transactions on Image Processing, vol. 24, no. 12, pp. 5826-5841, 2015.

13. Xiangyuan Lan, Shengping Zhang, and Pong C. Yuen, "Robust joint discriminative feature learning for visual tracking," in Proceedings of the Twenty-Fifth International Joint Conference on Artificial Intelligence. 2016, IJCAI'16, pp. 3403-3410, AAAI Press.

14. Xiangyuan Lan, Pong C. Yuen, and Rama Chellappa, "Robust mil-based feature template learning for object tracking," in AAAI, 2017.

15. Xiangyuan Lan, Mang Ye, Shengping Zhang, and Pong C. Yuen, "Robust collaborative discriminative learning for rgb-infrared tracking," in $A A A I, 2018$.

16. Elisa Ricci and Renzo Perfetti, "Retinal blood vessel segmentation using line operators and support vector classification," IEEE TRANSACTIONS ON MEDICAL IMAGING, vol. 26, no. 10, pp. 1357-1365, 2007.

17. Uyen T.V. Nguyen, Alauddin Bhuiyan, Laurence A.F. Park, and Kotagiri Ramamohanarao, "An effective retinal blood vessel segmentation method using multi-scale line detection," Pattern Recognition, vol. 46, pp. 703-715, 2013.

18. S. Roychowdhury, D. D. Koozekanani, and K. K. Parhi, "Blood vessel segmentation of fundus images by major vessel extraction and subimage classification," IEEE Journal of Biomedical and Health Informatics, vol. 19, no. 3, pp. 1118-1128, 2015.

19. M.M Fraz, P. Remagnin, A. Hoppe, B. Uyyanonvara, A.R. Rudnicka, C.G. Owen, and S.A. Barman, "Blood vessel segmentation methodologies in retinal images a survey," Computer Methods and Programs in Biomedicine, vol. 108, pp. 407-433, 2012.

20. D. Marin, A. Aquino, M. E. Gegundez-Arias, and J. M. Bravo, "A new supervised method for blood vessel segmentation in retinal images by using gray-level and moment invariants-based features," IEEE Transactions on Medical Imaging, vol. 30, no. 1, pp. 146-158, 2011.

21. "Hierarchical retinal blood vessel segmentation based on feature and ensemble learning," Neurocomputing, vol. 149, pp. 708 - 717, 2015.

22. P. Liskowski and K. Krawiec, "Segmenting retinal blood vessels with deep neural networks," IEEE Transactions on Medical Imaging, vol. 35, no. 11, pp. 2369-2380, 2016.

23. Q. Li, B. Feng, L. Xie, P. Liang, H. Zhang, and T. Wang, "A cross-modality learning approach for vessel segmentation in retinal images," IEEE Transactions on Medical Imaging, vol. 35, no. 1, pp. 109-118, 2016.
24. H. Fu, Y. Xu, D. W. K. Wong, and J. Liu, "Retinal vessel segmentation via deep learning network and fully-connected conditional random fields," in 2016 IEEE 13th International Symposium on Biomedical Imaging (ISBI), 2016, pp. 698-701.

25. Yanli Hou, "Automatic segmentation of retinal blood vessels based on improved multiscale line detection," Journal of Computing Science and Engineering, vol. 8, no. 2, pp. 119-128, 2014.

26. Niemeijer, Staal B, and van Ginneken, "Comparative study on retinal vessel segmentation methods on a new publicly available database.," SPIE, 2004.

27. Tony P. Pridmore, "Thresholding images of line drawings with hysteresis," in Fourth International Workshop on Graphics Recognition Algorithms and Applications, 2002, pp. 310-319.

28. A. Canny, J., "Computational approach to edge detection," IEEE Trans. Pattern Analysis and Machine Intelligence, vol. 8, no. 6, pp. 679-698, 1986.

29. M Kass and A Witkin, "Analyzing oriented patterns," Computer vision, graphics, and image processing, vol. 37 , no. 3, pp. 362385, 1987.

30. Q. Li, B. Feng, L. Xie, P. Liang, H. Zhang, and T. Wang, "A cross-modality learning approach for vessel segmentation in retinal images," IEEE Transactions on Medical Imaging, vol. 35, no. 1, pp. 109-118, 2015.

31. J. Staal, M. D. Abramoff, M. Niemeijer, M. A. Viergever, and B. van Ginneken, "Ridge-based vessel segmentation in color images of the retina," IEEE Transactions on Medical Imaging, vol. 23, no. 4, pp. 501-509, 2004.

32. Toufique Ahmed Soomro, Mohammad AU Khan, Junbin Gao, Tariq M Khan, and Manoranjan Paul, "Contrast normalization steps for increased sensitivity of a retinal image segmentation method," Signal, Image and Video Processing, vol. 11, no. 8, pp. 1509-1517, 2017.

33. Jose Ignacio Orlando and Matthew Blaschko, "Learning fullyconnected crfs for blood vessel segmentation in retinal images," Medical Image Computing and Computer Assisted Intervention (MICCAI),, vol. 17, pp. 634-641, 2014.

34. Toufique Ahmed Soomro, Mohammad AU Khan, Junbin Gao, Tariq Mahmood Khan, Manoranjan Paul, and Nighat Mir, "Automatic retinal vessel extraction algorithm.," in DICTA, 2016, pp. $1-8$.

35. Mohammad AU Khan, Toufique A Soomro, Tariq M Khan, Donald G Bailey, Junbin Gao, and Nighat Mir, "Automatic retinal vessel extraction algorithm based on contrast-sensitive schemes," in Image and Vision Computing New Zealand (IVCNZ), 2016 International Conference on. IEEE, 2016, pp. 1-5.

36. Joo V. B. Soares, Jorge J. G. Leandro, Roberto M. Cesar Jr, Herbert F. Jelinek, and Michael J. Cree, "Retinal vessel segmentation using the 2-d gabor wavelet and supervised classification," IEEE TRANSACTIONS ON MEDICAL IMAGING, 2006.

37. Carmen Alina Lupas, Domenico Tegolo, and Emanuele Trucco, "Fabc: Retinal vessel segmentation using adaboost," IEEE Transactions on Information Technology In Biomedicine, vol. 14, no. 5, pp. 1267-1274, 2010.

38. George Azzopardia, Nicola Strisciuglio, Mario Vento, and Nicolai Petkov, "Trainable cosfire filters for vessel delineation with application to retinal images," Medical Image Analysis, vol. 19, no. 1, pp. 46-57, 2015. 